\title{
Designing thickeners by matching hindered settling and gelled suspension zones in the presence of aggregate densification
}

\author{
Yi Zhang ${ }^{\mathrm{a}}$, Paul Grassia ${ }^{\mathrm{b}, *}$, Alastair Martin ${ }^{\mathrm{c}}$, Shane P. Usher ${ }^{\mathrm{d}}$, \\ Peter J. Scales ${ }^{\mathrm{d}}$ \\ ${ }^{a}$ CEAS, The Mill, University of Manchester, Oxford Road, Manchester M13 9PL, UK \\ ${ }^{b}$ Department of Chemical and Process Engineering, University of Strathclyde, \\ James Weir Building, 75 Montrose St, Glasgow G1 1XJ, UK \\ ${ }^{c}$ Department of Engineering, Lancaster University, Bailrigg, Lancaster LA1 4YR, UK \\ ${ }^{d}$ Department of Chemical and Biomolecular Engineering, University of Melbourne, \\ VIC 3010, Australia
}

\begin{abstract}
A model is presented for design of a thickener for solid-liquid separation where the flocs or aggregates within the solid-liquid suspension undergo an aggregate densification process due to the action of rakes. This aggregate densification facilitates suspension dewatering. The novel feature of the model is that it manages to couple together a hindered settling zone (higher up in the thickener, where the flocs are separated from one another, and the suspension cannot bear weight) and a gelled suspension zone (lower down in the thickener, where the flocs are packed together, and the suspension is able to bear weight). The model determines solids fraction profiles throughout the hindered settling zone and gelled suspension zone, and also gives zone heights
\end{abstract}

\footnotetext{
*Corresponding author

Email address: paul.grassia@strath.ac.uk (Paul Grassia)
} 
and residence times. Parametric investigations using the model are carried out for different suspension fluxes (which influence the solids fluxes and underflow solids fractions attained), and also for different specified amounts of and rates of aggregate densification.

Keywords:

Gels; Mathematical modelling; Rheology; Suspension; Compressive Yield Stress; Hindered Settling Function

\section{Highlights}

- A continuous thickener for solid-liquid separation is modelled

- Flocs within the thickener undergo aggregate densification facilitating dewatering

- Model couples hindered settling zone and gelled suspension zone within thickener

- Depending on operating regime, thickener height can be dominated by either zone

- Gelled zone dominates as suspension flux through thickener is reduced

\section{Introduction}

The need to dewater solid-liquid suspensions to reduce volumes of solidliquid waste and extract clean water is common in many industries (e.g. minerals processing (Boger, 2009; Jones and Boger, 2012), wastewater (Martin, 2004), dairy processing (Matsche et al., 2002), pulp and paper (Pere et al., 
1993)). Continuous thickeners are amongst the devices used for achieving such dewatering (Bustos et al., 1999).

Theories exist in literature for design of thickeners to achieve a given target solids flux and/or a given target underflow solids fraction (Usher and Scales, 2005; Diehl, 2001, 2008, 2012). Traditionally (Talmage and Fitch, 1955; Fitch, 1966) these have been based around Kynch theory (Kynch, 1952). This theory recognises (i) that solids settle due to buoyancy through being heavier than surrounding liquid, (ii) that buoyancy is balanced by viscous drag, and moreover (iii) that settling becomes hindered as the solids fraction in the suspension increases. Whilst the Kynch theory approach does indeed predict solids fluxes (and hence thickener cross sectional areas for a given volumetric flow rate of incoming suspension (Talmage and Fitch, 1955)), it does not predict the thickener heights.

Moreover one shortcoming of Kynch theory is that it tends to lose applicability as the solids fraction rises. Usually in dewatering applications one deliberately adds flocculants to the suspension, and these cause solid particles to aggregate together into flocs, with these flocs then settling faster than individual solid particles would (Heath et al., 2006). As the overall solids fraction becomes high enough however, the flocs can themselves network together into a weight bearing gel. Network stresses that oppose settling then develop in the gel (Buscall and White, 1987), meaning that buoyancy and viscous drag are no longer in balance.

Thickener design can then be achieved via Buscall and White theory (Bus- 
call and White, 1987; Landman et al., 1988; Bürger and Concha, 1998). This theory (unlike Kynch theory) is able to predict thickener heights (Usher and Scales, 2005), or more specifically it is able to predict the height of a consolidated bed of gelled suspension thereby setting a lower bound for the possible thickener height. The stronger the weight bearing strength of the gelled suspension, the taller the thickener must be.

Yet another complication one encounters during thickening is that the aggregates or flocs can change their structure in real time during the thickening process. This can happen as a result of the flocs being subjected to shear within a thickener, e.g. due to the action of rakes (Spehar et al., 2014; Gladman et al., 2010). Shear leads to so called aggregate densification, i.e. individual aggregates bind together more tightly (Farrow et al., 2000; Usher et al., 2009; van Deventer et al., 2011), which is highly beneficial for the dewatering process. Not only are wider channels opened up between flocs facilitating dewatering, but individual flocs also tend to lose contact with their neighbours, decreasing the suspension's weight bearing strength hence promoting consolidation (Usher et al., 2009; van Deventer et al., 2011).

Aggregate densification thereby allows a given thickener to achieve higher solids fluxes and/or higher underflow solids fractions than before, or alternatively allows redesign of a less tall thickener. Given the importance of aggregate densification, a number of studies have been dedicated to determining how to incorporate it into thickener design procedures (Usher et al., 2009; van Deventer et al., 2011; Zhang et al., 2013a,b). By and large these 
studies focussed on incorporating aggregate densification into the Buscall and White framework. Apart from the fact that aggregate densification is predicted to enhance thickener performance significantly, the approach is conceptually not very different from conventional Buscall and White theory.

Recently however a study has appeared (Grassia et al., 2014) suggesting how to incorporate aggregate densification into the framework of Kynch theory. This study would apply to situations where the degree of thickening required (measured by a target underflow solids fraction) is relatively modest so that either the underflow does not form a gel (Spehar et al., 2014) or else it is just barely gelled, with very significant amounts of ungelled material elsewhere in the thickener. Alternatively the study of Grassia et al. (2014) could be considered to correspond to a case where a particularly high solids flux is required (since there is known to be a trade-off between solids flux achieved and solids fraction attained during thickening (Usher and Scales, 2005)). In contrast to conventional Kynch theory (which as mentioned earlier does not predict thickener heights) this recent study (Grassia et al., 2014) combining aggregate densification with Kynch theory actually managed to make predictions for thickener heights. Specifically it predicted the height of a hindered settling zone, a region throughout which the suspension is not gelled. There still might be a consolidated bed of gelled suspension very close to the underflow, but Grassia et al. (2014) assumed this to be of negligible thickness compared to the hindered zone.

Even though the effects of aggregate densification have been considered 
upon 'ungelled' hindered settling zone heights (via Kynch theory (Grassia et al., 2014)) and upon gelled suspension bed heights (via Buscall and White theory (Usher et al., 2009; Zhang et al., 2013a,b)), to date there has never been a study which combines the two theories together so as to design a thickener subject to aggregate densification incorporating both a hindered settling zone and a consolidated bed each of which make a significant contribution to the overall height. Indeed it is not even clear that, in the presence of aggregate densification, it is always feasible to combine the two theories together. Establishing under what conditions the theories can be combined, and performing design calculations using the combined theories are the topics of the present work.

This study is laid out as follows. Section 2 details the separate theories of thickener design incorporating aggregate densification (Kynch vs Buscall and White), after which section 3 explains how to match those theories together: matching involves comparing the solids fraction that Kynch theory determines in the hindered settling zone to the solids fraction at which the suspension starts to form into a weight bearing gel (the top of the gelled suspension zone according to Buscall and White theory). A number of case studies are set up in section 4, and results from the case studies are presented in section 5. Finally conclusions are offered in section 6 . 


\section{Theory}

This section is laid out in three parts. The first part (section 2.1) describes thickener design using Kynch theory, and in particular how aggregate densification modifies the theory: the reader is referred to Grassia et al. (2014) for details. The second part (section 2.2) is analogous but focusses on Buscall and White theory with aggregate densification: the reader can refer to Usher et al. (2009); van Deventer et al. (2011); Zhang et al. (2013a,b) for full details. The third part (section 2.3) explains how to cast the system in dimensionless form, indicating which dimensionless scalings are most useful for which zone of the thickener.

\subsection{Kynch theory in the presence of aggregate densification}

The key element of Kynch theory (Kynch, 1952) (that applies to ungelled suspensions) is a material property of the suspension called the hindered settling function (Usher and Scales, 2005; Lester et al., 2005). This governs how the effective frictional force on settling solids increases as a function of solids fraction. This hindered settling function is denoted by $R(\phi)$ where $\phi$ is the solids volume fraction. Usually $R(\phi)$ is a sharply increasing function of $\phi$ (Grassia et al., 2008).

According to Kynch theory, the batch settling or 'free settling' flux of 
solids (denoted $q_{f s}$ ) is related ${ }^{1}$ to $R(\phi)$ via (Lester et al., 2005)

$$
q_{f s}=\frac{\Delta \rho g \phi(1-\phi)^{2}}{R(\phi)}
$$

where $\Delta \rho$ is the density difference between solid and liquid (assumed to equal $2200 \mathrm{~kg} \mathrm{~m}^{-3}$ here), and where $g$ is acceleration due to gravity $\left(9.8 \mathrm{~m} \mathrm{~s}^{-2}\right)$. In the case of a thickener there is a suspension flux $q_{s}$ flowing through the thickener in addition to settling of solids, and as a result the total underflow flux of solids $q_{u}$ becomes

$$
q_{u}=q_{s} \phi+q_{f s}
$$

and where moreover the underflow solids fraction $\phi_{u}$ is $q_{u} / q_{s}$. Usually design proceeds (Diehl, 2001, 2008; Grassia et al., 2014) by first selecting $q_{s}$, and then finding a target $q_{u}$ that corresponds to a local minimum of equation (2) with respect to $\phi$. That the right hand side of equation (2) can admit a local minimum follows from the functional form of $q_{f s}$ in equation (1). This vanishes for $\phi=0$, then grows as $\phi$ increases, but starts to decrease again owing to the very sharp increases in $R(\phi)$. In the domain where $q_{f s}$ is decreasing, if $\mathrm{d} q_{f s} / \mathrm{d} \phi$ happens to attain the value $-q_{s}$, then a necessary condition is met for a local minimum in equation (2).

Now consideration must be given as to how the presence of aggregates

\footnotetext{
${ }^{1}$ The $(1-\phi)^{2}$ term in the numerator of equation (1) arises from the solids batch settling speed being less than the solid-to-liquid velocity difference, and the pressure field in the liquid providing more upthrust than a purely hydrostatic field would.
} 
(i.e. flocs) affects the above picture. It is supposed (Usher et al., 2009) that aggregates have a solids fraction $\phi_{a g g}$ that in general differs from the (overall) solids fraction $\phi$. The ratio $\phi / \phi_{\text {agg }}$ represents the fraction of space filled by flocs. In the case of interest in the current section, i.e. ungelled suspensions, flocs must be not in contact with one another so $\phi / \phi_{\text {agg }}$ must be strictly less than a close packing fraction (that following Usher et al. (2009); Zhang et al. (2015) is assumed to equal 0.6).

In the presence of aggregate densification, $\phi_{a g g}$ becomes a function of the residence time $t_{\text {res }}$ that flocs spend in the system. Following the theory of Usher et al. (2009); van Deventer et al. (2011) first aggregate diameters (denoted $d_{a g g}$ ) are determined, and these are then related to $\phi_{\text {agg }}$. Specifically it is assumed that during densification aggregate diameters $d_{\text {agg }}$ follow an empirical law

$$
d_{\text {agg }}=d_{\text {agg }, \infty}+\left(d_{\text {agg }, 0}-d_{\text {agg }, \infty}\right) \exp \left(-A t_{r e s}\right)
$$

where $d_{a g g, 0}$ is the 'undensified' diameter, $d_{a g g, \infty}$ is the 'fully densified' diameter $^{2}$, and $A$ is a densification rate parameter. In cases where aggregate densification is produced by raking the suspension, $A$ depends on the rate of raking. Potentially aggregate densification could also be sensitive to solids concentration (Spehar et al., 2014), and since solids concentration varies with

\footnotetext{
${ }^{26}$ Fully densified' does not imply that the solids in the aggregate are themselves close packed. It merely means that the aggregate diameter has ceased to change with time even though the aggregate continues to be subject to shear.
} 
position in the thickener, and since position reached depends on residence time $t_{\text {res }}$, potentially $A$ could become a function of $t_{\text {res }}$ : such complications will however be neglected here. Following literature (van Deventer et al., 2011; Zhang et al., 2013a) it is assumed $d_{a g g, \infty} / d_{a g g, 0}=0.9$ and (in what follows) two different values of $A$ are also considered, namely $A=0.001 \mathrm{~s}^{-1}$ and $A=0.01 \mathrm{~s}^{-1}$. Conservation of mass of solids in each aggregate whilst the aggregates densify implies

$$
\phi_{\text {agg }}=\phi_{\text {agg }, 0} d_{\text {agg, } 0}^{3} / d_{a g g}^{3}
$$

where $\phi_{a g g, 0}$ is the initial 'undensified' solids fraction in the aggregates that is taken as in Usher et al. (2009) to equal 0.1667.

The aggregate densification theory of Usher et al. (2009) explains how densification causes wider channels to be opened up between aggregates which results in lower frictional drag and faster aggregate settling. The hindered settling function $R$ now becomes a function of both $\phi$ and $\phi_{\text {agg }}$. In particular, at any given $\phi$, the function $R$ decreases as $\phi_{\text {agg }}$ increases. The theory then specifies how to relate a densified hindered settling function $R$ to an undensified one (which is denoted $R_{0}$ ).

Following van Deventer et al. (2011), a functional form for $R_{0}$ representative of a minerals tailings slurry is adopted

$$
R_{0}=\frac{R_{\text {Stokes }, 0}}{\phi_{\text {agg }, 0}}\left(\phi+r_{g}\right)^{r_{n}} r_{g}^{-r_{n}}
$$


where $R_{\text {Stokes }, 0}$ is the initially undensified hindered settling function of an isolated aggregate or floc, and $r_{g}$ and $r_{n}$ are fitting parameters. The settling speed of the isolated aggregate or floc (denoted $\left.u_{\text {Stokes }, 0}\right)$ is (by definition) $\Delta \rho g / \lim _{\phi \rightarrow 0} R_{0}$ where $\Delta \rho$ is the density difference between solids and liquid, and where $g$ is gravitational acceleration, the above definition applying even though the floc itself is not entirely composed of solids. Note that this result for an isolated floc can also be written $\phi_{a g g, 0} \Delta \rho g / R_{\text {Stokes }, 0}$, the factor $\phi_{a g g, 0}$ in the numerator reflecting that the effective density difference between a floc and the liquid is less than the solid-liquid density difference. Values are assigned as (Zhang et al., 2013a,b) $R_{\text {Stokes }, 0}=260469 \mathrm{Pas} \mathrm{m}^{-2}, r_{g}=0.05$, $r_{n}=5$, with in addition $\phi_{a g g, 0}=0.1667$ as mentioned earlier. The theory then gives (Usher et al., 2009)

$$
\begin{gathered}
R=\frac{(1-\phi)^{2} R_{0}\left(\phi_{\text {agg }}\right) R_{\text {Stokes }, 0} D_{\text {agg }}}{\left(\phi_{\text {agg }, 0} R_{0}\left(\phi_{\text {agg }}\right)\left(1-\phi / \phi_{\text {agg }}\right)^{2} / r_{\text {agg }}\left(\phi / \phi_{\text {agg }}\right)+\right.} \\
\left.\left(R_{\text {Stokes }, 0} D_{\text {agg }} \phi / \phi_{\text {agg }}\right)\left(1-\phi_{\text {agg }}\right)^{2}\right)
\end{gathered}
$$

where $D_{a g g}$ is the ratio $d_{a g g} / d_{a g g, 0}$ and where $r_{a g g}$ is the aggregate hindered settling factor (a function of $\phi / \phi_{a g g}$ ) given by

$$
\begin{gathered}
r_{\text {agg }}=\frac{\phi_{\text {agg }, 0}\left(1-\phi / \phi_{\text {agg }}\right)^{2} R_{0}\left(\phi_{\text {agg }, 0}\right) R_{0}\left(\phi_{\text {agg }, 0} \phi / \phi_{a g g}\right)}{R_{\text {Stokes }, 0}\left(R_{0}\left(\phi_{\text {agg }, 0}\right)\left(1-\phi_{\text {agg }, 0} \phi / \phi_{\text {agg }}\right)^{2}-\right.} \\
\left.\phi \phi_{\text {agg }}^{-1}\left(1-\phi_{\text {agg }, 0}\right)^{2} R_{0}\left(\phi_{\text {agg }, 0} \phi / \phi_{\text {agg }}\right)\right)
\end{gathered}
$$

Graphs of $R$ vs $\phi$ (or more precisely of a dimensionless quantity $R_{s}$ de- 
fined as $R / R_{\text {Stokes }, 0}$ vs $\phi$ ) in the undensified and fully densified state are given in Figure 1 (note the log scale on the vertical axis). It is clear that the $R$ vs $\phi$ curves have the expected properties, namely $R$ is a rapidly increasing function of $\phi$ and the densified $R$ vs $\phi$ is less than the undensified one. Note that in the limit as $\phi \rightarrow 0$, the hindered settling function $R$ evaluates to $D_{\text {agg }} R_{\text {Stokes }, 0} / \phi_{\text {agg }, 0}$, the leading factor $D_{\text {agg }}$ reflecting the fact that the frictional drag on an isolated aggregate is reduced if its diameter decreases owing to densification, whilst the buoyancy force is unchanged (the mass of solids in the aggregate being conserved). Away from the $\phi \rightarrow 0$ limit, reductions in $R$ due to aggregate densification can be even more substantial than just the $D_{\text {agg }}$ factor referred to above: in fact the main effect (Grassia et al., 2014; van Deventer et al., 2011) upon equation (6) is a large reduction in the value of the term $r_{a g g}$, which according to equation (7) involves evaluating $R_{0}$ at an argument $\phi_{a g g, 0} \phi / \phi_{a g g}$, instead of at $\phi$. Since $R_{0}$ is a function that, according to equation (5), decreases rapidly as its argument decreases, even modest decreases in the ratio $\phi_{a g g, 0} / \phi_{\text {agg }}$ can lead to large decreases in $R_{0}\left(\phi_{a g g, 0} \phi / \phi_{a g g}\right)$, and hence large decreases in $r_{a g g}$ and $R$.

These decreases in $R$ at any given $\phi$ (in response to increasing amounts of aggregate densification) would clearly lead to increases in $q_{f s}$ and $q_{u}$ according to equations (1)-(2). The procedure developed in Grassia et al. (2014) was to suppose instead that $\phi$ must change in response to aggregate densification, in order to keep $q_{u}$ spatially uniform across a hindered settling zone: this gives a profile of $\phi$ values across the hindered settling zone where it is necessary 
to choose a branch of solutions such that $\phi$ increases with increasing depth. One still sets the $q_{u}$ value by minimising equation (2) but this minimisation is applied only at one point, namely the bottom of the hindered settling zone (Grassia et al., 2014) with $t_{\text {res }}$ in equation (3) now denoting the residence time spent traversing that zone.

\subsection{Buscall and White theory in the presence of aggregate densification}

Buscall and White theory requires, as well as the hindered settling function discussed above, an additional material property known as the compressive yield stress (Buscall and White, 1987; Usher and Scales, 2005). This is a measure of the compressive load that needs to be placed on a suspension to make it start consolidating. It is denoted by $P_{y}(\phi)$.

It is possible moreover (Landman and White, 1994) to identify a particular solids fraction called the gel point that is denoted $\phi_{g}$. If $\phi<\phi_{g}$ the suspension is ungelled, and $P_{y}(\phi)$ is (by definition) identically zero. If $\phi>\phi_{g}$ however, $P_{y}$ is greater than zero, and moreover $P_{y}$ is a rapidly increasing function of $\phi$.

In the presence of aggregate densification (Usher et al., 2009; van Deventer et al., 2011), $P_{y}$ becomes sensitive to the values of both $\phi$ and $\phi_{\text {agg }}$. Tighter binding of individual aggregates (i.e. an increase in $\phi_{\text {agg }}$ ) implies less contact between aggregates and hence less compressive strength of the suspension

(i.e. lower $P_{y}$ ). Moreover an increase in $\phi_{a g g}$ implies an increase in $\phi_{g}$ and hence a wider domain of $\phi$ values for which $P_{y}(\phi)$ is identically zero. This is 
because the ratio $\phi_{g} / \phi_{\text {agg }}$ is assumed to be fixed and to correspond to a close packing fraction 0.6 as mentioned earlier.

Following literature (van Deventer et al., 2011) a functional form is chosen for the undensified compressive yield stress to be typical of a minerals tailings slurry. This undensified compressive yield stress is denoted by $P_{y, 0}(\phi)$ and selected as

$$
P_{y, 0}=\frac{a_{0}\left(\phi-\phi_{g, 0}\right)}{\left(m+\phi-\phi_{g, 0}\right)\left(\phi_{c p}-\phi\right)^{n_{0}}}
$$

where (Zhang et al., 2013a, 2015) $a_{0}=3.7914 \mathrm{~Pa}, m=0.0363, n_{0}=10.8302$ and $\phi_{c p}=0.8$. Moreover $\phi_{g, 0}=0.1$ is the undensified gel point: observe that this is 0.6 times $\phi_{a g g, 0}$.

The densified functional form is chosen (Zhang et al., 2013a) to have a similar functional form to the one given above

$$
P_{y}=P_{y, 0}\left(\phi_{a g g}\right) \frac{\left(m+\phi_{a g g}-\phi_{g}\right)}{\left(m+\phi-\phi_{g}\right)} \frac{\left(\phi-\phi_{g}\right)}{\left(\phi_{a g g}-\phi_{g}\right)}\left(\frac{\phi_{c p}-\phi_{a g g}}{\phi_{c p}-\phi}\right)^{n_{1}}
$$

where the densified gel point $\phi_{g}$ is $\phi_{g, 0} d_{a g g, 0}^{3} / d_{a g g}^{3}$ (with $d_{a g g, 0}$ and $d_{a g g}$ being respectively densified and undensified aggregate diameters) and where

$$
n_{1}=\left(\phi_{c p}-\phi_{a g g}\right)\left(\frac{P_{y, 0}^{\prime}\left(\phi_{a g g}\right)}{P_{y, 0}\left(\phi_{a g g}\right)}+\frac{1}{m+\phi_{a g g}-\phi_{g}}-\frac{1}{\phi_{a g g}-\phi_{g}}\right) .
$$

Here $P_{y, 0}^{\prime}\left(\phi_{a g g}\right)$ is the derivative of $P_{y, 0}$ with respect to $\phi$ evaluated at $\phi_{a g g}$.

Plots are given of the undensified and fully densified compressive yield stress (or more precisely of a dimensionless quantity $p_{y}$ defined as $P_{y} / a_{0}$ ) 
in Figure 2. The expected features are observed, i.e. rapid increase in $P_{y}$ with increasing $\phi$, and also the densified $P_{y}$ being less than the undensified one. Similar comments apply to $P_{y}$ as already applied to $R$, i.e. even a comparatively modest change in $\phi_{\text {agg }}$ (and consequently in $\phi_{g}$ which is directly proportional to $\phi_{\text {agg }}$ ) can lead to large relative changes in $P_{y}$. This is particularly apparent for $\phi$ values which are just very slightly above the densified $\phi_{g}$ (leading to a very tiny $P_{y}$ ) but which are substantially above $\phi_{g, 0}$ (and hence having a substantial $P_{y, 0}$ value).

The equation used to design thickeners according to Buscall and White theory with aggregate densification is as follows (Zhang et al., 2013a)

$$
\frac{\mathrm{d} \phi}{\mathrm{d} t_{\text {res }}}=\frac{\Delta \rho g q_{u}-(1-\phi)^{-2} R\left(\phi, t_{r e s}\right) q_{u}^{2}\left(\phi^{-1}-\phi_{u}^{-1}\right)-\partial P_{y}\left(\phi, t_{r e s}\right) / \partial t_{r e s}}{\partial P_{y}\left(\phi, t_{r e s}\right) / \partial \phi}
$$

and corresponds to a balance between buoyancy, viscous drag and network stresses: the presence of network stresses in Buscall and White theory implies that solids flux is no longer given by the simple Kynch theory form in equation (2). In the above $\phi_{u}$ is the underflow solids fraction and $q_{u}$ is the underflow solids flux, and these are related to the suspension flux $q_{s}$ via $\phi_{u} \equiv q_{u} / q_{s}$. Moreover $R$ and $P_{y}$ have been expressed as functions of $\phi$ and $t_{r e s}$ instead of as functions of $\phi$ and $\phi_{a g g}$, recognising that $\phi_{a g g}$ is itself a function of $t_{\text {res }}$ (according to the theory presented in section 2.1). Aggregate densification then impacts on thickener design via the strong sensitivity of both $R$ and $P_{y}$ to the level of aggregate densification or equivalently their 
strong sensitivity to $t_{\text {res }}$.

Buscall and White theory (unlike Kynch theory) does not invariably fix $q_{u}$ for a given $q_{s}$ via a local minimization of equation (2) since network stresses may affect the solids settling rate. In fact, thickener control systems typically select a specified underflow rheology (which correlates with a specific $\phi_{u}$ ) so that $q_{u}$ is then obtained for any given $q_{s}$. Equation (11) can then be used to generate a profile of $\phi$ vs vertical coordinate $z$ recognizing that

$$
\mathrm{d} z / \mathrm{d} t_{r e s}=-q_{u} / \phi
$$

The negative sign here reflects the convention that $z$ is measured upwards, whereas solids settle downwards.

Although equation (11) applies to a system undergoing aggregate densification, the special case of either an undensified system or a system that has already reached full densification is simple to obtain: one simply discards the $\partial P_{y} / \partial t_{\text {res }}$ term from the numerator on the right hand side of equation (11). Such systems have been studied by Usher et al. (2009); Zhang et al. (2013b).

\subsection{Converting to dimensionless form}

In order to perform further analysis on thickener design, it is convenient to cast the system in dimensionless form. The velocity scale $u_{\text {Stokes }, 0}$ is defined to be $\Delta \rho g / R_{\text {Stokes }, 0}$ (evaluating to $0.08277 \mathrm{~m} \mathrm{~s}^{-1}$ for the parameter values quoted in section 2.1) and fluxes $q_{u}, q_{s}$ and $q_{f s}$ are made dimensionless on the scale $u_{\text {Stokes }, 0}$. Dimensionless fluxes are denoted by the symbols $Q_{u}, Q_{s}$ 
and $Q_{f s}$. Residence times $t_{\text {res }}$ are made dimensionless on the scale $A^{-1}$ (the reciprocal of the densification rate parameter) and distances $z$ are made dimensionless on the scale $u_{\text {Stokes }, 0} / A$. Interest here focuses on values either $A=0.001 \mathrm{~s}^{-1}$ or $A=0.01 \mathrm{~s}^{-1}$. In the latter case, 1 unit of dimensionless time corresponds to $100 \mathrm{~s}$ and 1 unit of dimensionless distance corresponds to $8.277 \mathrm{~m}$. In the former case however, the unit of dimensionless distance and time correspond to $1000 \mathrm{~s}$ and $82.77 \mathrm{~m}$ respectively. This does not imply that thickeners must be designed to be anywhere near $82.77 \mathrm{~m}$ tall, since it can happen that the predicted thickener height is found to be just a small fraction of 1 dimensionless height unit. In what follows, the symbol $T_{\text {res }}$ denotes the dimensionless residence time and $Z$ denotes the dimensionless distance coordinate. It is a straightforward matter to re-express all the governing equations from sections 2.1-2.2 in dimensionless form, although in the interests of brevity the resulting equations are not reproduced here. Implications of using the non-dimensional equations are however discussed below.

Recall that in a thickener one typically has a hindered settling zone above and a gelled suspension zone below. The dimensionless scales quoted above collapse the data in the hindered settling zone together. Specifically if there are two systems with different densification rate parameters $A$ (corresponding to different rates of raking the suspension), but with identical suspension fluxes, and with the same overall amount of densification achieved in the hindered zone, then the two systems pass through the exact same sequence of $\phi$ and $\phi_{\text {agg }}$ states in the hindered settling zone (albeit, when converting 
back to dimensional variables, at different rates according to the value of $A$ ).

Dimensionless data corresponding to these two different $A$ values does not however continue to collapse once the gelled suspension bed is reached. Indeed it is possible to define a dimensionless analogue of the densification rate parameter

$$
\alpha=\frac{a_{0} A}{\Delta \rho g u_{\text {Stokes }, 0}}
$$

and, the state of the gelled suspension (in particular $\phi$ expressed in terms of dimensionless $T_{\text {res }}$ ) becomes sensitive to $\alpha$. For the parameter values quoted in sections 2.1-2.2 it is found that ${ }^{3} \alpha=2.125 \times 10^{-6}$ (if $A=0.001 \mathrm{~s}^{-1}$ ) and $\alpha=2.125 \times 10^{-5}\left(\right.$ if $\left.A=0.01 \mathrm{~s}^{-1}\right)$.

There is an alternative scaling that can be applied in the gelled suspension bed. If one divides the dimensionless distance $Z$ by $\alpha$, this is equivalent to making the distance $z$ dimensionless on the scale $a_{0} /(\Delta \rho g)$ instead of $u_{\text {Stokes }, 0} / A$. Likewise if one divides the dimensionless time $T_{\text {res }}$ by $\alpha$ this is equivalent to making the time $t_{\text {res }}$ dimensionless on the scale $a_{0} /\left(\Delta \rho g u_{\text {Stokes }, 0}\right)$ instead of $A^{-1}$. These new scales $a_{0} /(\Delta \rho g)$ for distance and $a_{0} /\left(\Delta \rho g u_{\text {Stokes }, 0}\right)$ for time (evaluating for the data considered here to $2.125 \times 10^{-3} \mathrm{~s}$ and $1.758 \times 10^{-4} \mathrm{~m}$ ) are both independent of the densification rate parameter $A$, making them particularly convenient for analysing how

\footnotetext{
${ }^{3}$ Although the dimensionless densification rate $\alpha$ values are much smaller than unity here, this does not mean that densification rates are irrelevant to the thickening process. Since $P_{y}$ and $R$ are rapidly increasing functions of $\phi$, it follows that $a_{0}$ in equation (13) is an underestimate of typical compressive stress in a finite solids fraction suspension, and likewise $u_{\text {Stokes }, 0}$ in equation (13) is an overestimate of the typical settling velocity.
} 
gelled bed heights and residence times in the gelled bed are sensitive to $A$ (or equivalently to $\alpha$ ). It is not the case that residence times in the gelled bed will be anywhere near as small as $2.125 \times 10^{-3} \mathrm{~s}$, nor will bed heights be anywhere near as short as $1.758 \times 10^{-4} \mathrm{~m}$, because the times and heights that are computed during thickener design turn out to be many multiples of these rescaled units.

In summary one set of dimensionless variables is convenient for collapsing data in the hindered settling zone, whilst a distinct set of dimensionless variables is more convenient for interpreting data in the gelled suspension zone. Accordingly, both sets of variables will be considered (as appropriate) in what follows.

\section{Technique for matching hindered and consolidated zones}

Sections 2.1 and 2.2 considered respectively how to model the hindered settling zone and consolidated bed zones separately. The challenge now is to match those two zones together. This is achieved below by comparing the solids fraction at the bottom of the hindered zone and the solids fraction at the top of the gelled suspension bed. Matching is in fact achieved by constraining the first of these solids fractions to be less than the second.

It has been shown by Grassia et al. (2014) that the most convenient way of describing the hindered zone is to parameterise in terms of the residence time $T_{\text {res }}$ that is spent traversing that zone (and using this to determine the height of the hindered settling zone). This was found to be far more 
straightforward than trying to fix the height of the hindered zone a priori, and then attempting to find the $T_{\text {res }}$ value that produced that target height. It turns out that parameterising in terms of residence time $T_{r e s}$ that is spent in the hindered zone, is actually also the most convenient way of matching the hindered zone and gelled suspension zone together, so this parameterisation is pursued further in what follows. As we shall see, in certain cases, the solids fraction at the bottom of the hindered zone remains less than the suspension gel point at the top of the bed, regardless of the value of $T_{r e s}$ chosen. In other cases however, to keep the first of these solids fractions less than the second, an upper limit must be imposed on the time $T_{\text {res }}$ that solids are allowed to spend in the hindered zone. The criteria determining that $T_{r e s}$ is unrestricted in some cases and yet restricted in others are explained below.

For any given $T_{\text {res }}$ and any given suspension flux $Q_{s}$ it is possible to construct a solids flux curve $Q_{u}$ vs $\phi$. Specifically (taking the dimensionless analogue of equation (2))

$$
Q_{u}=Q_{s} \phi+Q_{f s}
$$

where $Q_{f s}$ is the batch settling or 'free settling' flux, a well defined function of $\phi$. The function $Q_{f s}$ (analogously to $q_{f s}$ ) vanishes when $\phi \rightarrow 0$, and rises to a maximum as $\phi$ grows, but then decays very rapidly back towards zero as $\phi$ grows further still.

Typical $Q_{u}$ vs $\phi$ plots for a variety of $T_{\text {res }}$ values are shown in Figure 3 
for the arbitrarily chosen value $Q_{s}=0.0034$. As can be seen in Figure 3, it is generally possible to find a solids fraction $\phi_{\text {loc min }}$ that corresponds to a (local) minimum of the $Q_{u}$ vs $\phi$ curve at any given $T_{\text {res }}$. Note that $\phi_{\text {loc min }}$ is sensitive to the suspension flux $Q_{s}$ that is chosen. Had a $Q_{s}$ value smaller than 0.0034 been selected, the $\phi_{\text {loc min }}$ values obtained would have been larger: as is well known in the literature (Usher and Scales, 2005), there is a trade off between the flux through a thickener and the solids fractions attained.

The claim made above is that it is possible to match the hindered settling and gelled suspension zones of the thickener together (accounting for aggregate densification occuring in both zones) provided $\phi_{\text {loc min }}$ (the solids fraction at the bottom of the hindered zone) is less than the suspension gel point $\phi_{g}$ (which by definition is the solids fraction at the top of the gelled bed). By contrast situations where $\phi_{l o c}$ min exceeds $\phi_{g}$ lead to a contradiction: under those circumstances one should not use the Kynch theory of Grassia et al. (2014) to design for solids fractions up to $\phi_{l o c}$ min in the hindered zone, because material at $\phi_{l o c}$ min should already be in the gelled zone (and so the entire design should have employed the Buscall and White theory of Zhang et al. (2013a)).

Determining whether $\phi_{l o c \text { min }}$ is less or greater than $\phi_{g}$ depends partly on the suspension flux $Q_{s}$ that is proposed: as was mentioned above, decreasing $Q_{s}$ tends to increase $\phi_{l o c}$ min , thereby making it more likely to exceed $\phi_{g}$. However determination of the relation between $\phi_{\text {loc min }}$ and $\phi_{g}$ is further complicated by the fact that both $\phi_{l o c}$ min and $\phi_{g}$ are both functions of $T_{\text {res }}$. 
Understanding the variation of $\phi_{g}$ with respect to $T_{\text {res }}$ is straightforward. As aggregate densification occurs and individual aggregates bind more tightly together (increasing $\phi_{\text {agg }}$ ) it has already been mentioned that the ratio between $\phi_{g}$ (the gel point) and $\phi_{a g g}$ (the solids fraction in the aggregates) remains fixed. However Figure 3 makes it clear that also $\phi_{l o c}$ min increases with $T_{\text {res }}$ : in that figure, not only does the value of the local minimum increase with increasing $T_{\text {res }}$ (leading to increased solids fluxes $Q_{u}$, an expected beneficial outcome of aggregate densification) but also the $\phi$ value corresponding to that local minimum shifts to the right with increasing $T_{\text {res }}$. The value of $T_{\text {res }}$ then potentially affects the relation between $\phi_{l o c}$ min and $\phi_{g}$.

Three separate situations are now identified, with a discussion of how to design thickeners in each situation. In the first situation $\phi_{l o c} \min$ in the fully densified state is less than $\phi_{g}$ in the fully densified state (and indeed $\phi_{\text {loc min }}$ at any value of $T_{\text {res }}$ is less than $\phi_{g}$ at that same $T_{\text {res }}$ ). In the second situation $\phi_{\text {loc min }}$ in the fully densified state is greater than $\phi_{g}$ in the fully densified state, whereas $\phi_{l o c}$ min in the undensified state is less than $\phi_{g}$ in the undensified state. In the third situation $\phi_{l o c}$ min in the undensified state exceeds $\phi_{g}$ in the undensified state (and in fact $\phi_{l o c}$ min at any chosen $T_{\text {res }}$ values exceeds $\phi_{g}$ at that same $\left.T_{\text {res }}\right)$.

If $\phi_{\text {loc min }}<\phi_{g}$ in the fully densified state, one is free to choose any value of $T_{\text {res }}$, and having chosen that $T_{\text {res }}$, the hindered settling zone can be computed according to the procedures of section 2.1, assuming the suspension flux $Q_{s}$ is also given. At the bottom of the hindered zone, the solids fraction 
jumps from the ungelled $\phi_{\text {loc min }}$ to the gelled $\phi_{g}$ at the chosen $T_{\text {res }}$. The gelled suspension zone can then be computed following the procedures of section 2.2. Note that the solids flux $Q_{u}$ that is delivered and the underflow solids flux $\phi_{u}$ (which is simply $Q_{u} / Q_{s}$ ) are actually fixed by the hindered zone.

If the undensified $\phi_{l o c \min }<\phi_{g}$ but the fully densified $\phi_{l o c \min }>\phi_{g}$, there is then a critical residence time in the hindered zone at which $\phi_{l o c}$ min matches $\phi_{g}$. The computations that must be performed are analogous to the first situation described above, except that the $T_{\text {res }}$ values are constrained: specifically $T_{\text {res }}$ must be chosen less than the critical residence time described above, so that the jump to the gelled state cannot be delayed indefinitely. It is still however the case that $Q_{u}$ and $\phi_{u}$ are fixed by the hindered zone.

If the undensified $\phi_{\text {loc min }}>\phi_{g}$, the suspension can be considered to gel immediately, without the need to compute a hindered settling zone. Such situations have already been considered at length by Zhang et al. (2013a) and so are not studied any further here. The solids flux $Q_{u}$ through the system is no longer set by the hindered settling zone, but instead (Zhang et al., 2013a) can take any value in a range between zero and the value that equation (14) would predict in the undensified state at the undensified gel point: the higher the flux chosen, the taller the thickener.

In what follows a number of thickener design case studies are proposed corresponding to the first two of the situations mentioned above, permitting matching between the hindered settling zone and gelled suspension zone in 
the presence of aggregate densification.

\section{Case studies}

In this section, eight cases are illustrated (summarised in Table 1). Suspension material properties are all as given in section 2. Two distinct suspension fluxes are chosen: $Q_{s}=0.0034$ (the same value as is used as Figure 3, and corresponding to Cases 1-4) and a smaller suspension flux $Q_{s}=0.0015$ (corresponding to Cases 5-8). Two distinct residence times (in the hindered settling zone) are also chosen $T_{\text {res }}=0.05$ (Cases $1-2$ and 5-6) and $T_{\text {res }}=0.3$ (Cases 3-4 and 7-8).

Odd and even numbered cases can be distinguished according to the densification rate parameter, odd numbered cases have $A=0.001 \mathrm{~s}^{-1}$ (equivalently $\alpha=2.125 \times 10^{-6}$ ) whilst even numbered cases have $A=0.01 \mathrm{~s}^{-1}$ (equivalently $\alpha=2.125 \times 10^{-5}$ ). Remember however that (owing to the dimensionless scalings employed that collapse data together in the hindered settling zone) the distinction between odd and even numbered cases will only become apparent when consideration is given to the state of the gelled suspension bed.

In the systems of interest, it is the hindered settling zone (not the gelled bed) that sets the solids flux $Q_{u}$, and this flux can be determined immediately, with values tabulated in Table 1. Moreover the underflow solids volume fraction $\phi_{u}$ can also be obtained: it is just $Q_{u} / Q_{s}$. The data in Table 1 bear out the expectation that smaller $Q_{s}$ values produce larger solids fractions $\phi_{u}$.

Values of $\phi_{\text {loc min }}$ (the solids fraction at the bottom of the hindered settling 
zone, as opposed to that in the thickener underflow) are likewise shown in Table 1 and exhibit similar trends, i.e. smaller $Q_{s}$ gives larger $\phi_{l o c}$ min.

Table 1 compares $\phi_{l o c}$ min values with gel points $\phi_{g}$ : recall that $\phi_{l o c}$ min must be less than $\phi_{g}$ in order to match the hindered and gelled zones together. It is instructive to plot $\phi_{l o c \min }$ and $\phi_{g}$ as functions of $T_{r e s}$ and this is done in Figure 4a (for $\left.Q_{s}=0.0034\right)$ and in Figure $4 \mathrm{~b}$ (for $\left.Q_{s}=0.0015\right)$. It is apparent that if $Q_{s}=0.0034$, then $\phi_{\text {loc min }}<\phi_{g}$ for all $T_{\text {res }}$, whereas if $Q_{s}=0.0015$, then this only applies up to $T_{\text {res }} \approx 0.378$. In all of Cases $1-8$ at least, the constraint that $\phi_{l o c \min }<\phi_{g}$ is met. It is possible therefore to use the techniques of Grassia et al. (2014) and Zhang et al. (2013a) to compute profiles of solids fraction $\phi$ vs $Z$ respectively in the hindered settling zone and the gelled suspension zone, and compare the heights of and amounts of time spent in each zone. This is achieved in the next section.

\section{Results}

This results section is arranged in three parts. Section 5.1 considers profiles of solids fraction vs position in the hindered settling zone. Then section 5.2 analyses profiles of solids fraction vs position in the gelled suspension zone. Finally section 5.3 considers residence times and zone heights for both the hindered settling and gelled suspension zones.

\subsection{Solids fraction profiles in the hindered zone}

Solids fraction profiles across the hindered settling region are plotted in Figure 5. The convention adopted is to measure $Z$ upwards, and to place 
$Z=0$ at the top of the hindered settling region, so interest focusses upon values $Z \leq 0$ here. The solids fraction $\phi$ increases downwards through the hindered settling zone as was also seen in Grassia et al. (2014).

Observing Figure 5, the main trend seen is that the size of the hindered settling region is smaller with smaller residence time $T_{\text {res }}$ (e.g. Cases $1-2$ vs Cases 3-4, Cases 5-6 vs Cases 7-8). Yet another trend is that cases with higher $\phi$ values (through selecting a lower $Q_{s}$ ) have a smaller hindered settling region because the settling speed is slower (e.g. Cases 5-8 vs Cases 1-4).

\subsection{Solids fraction profiles in the gelled suspension zone}

Solids fraction profiles in the gelled suspension region are shown in Figure 6 and Figure 7. Here a shifted and rescaled dimensionless coordinate $Z^{\prime}$ is used, defined as $Z^{\prime}=\left(Z-Z_{h}\right) / \alpha$ where $Z_{h}$ denotes the vertical coordinate (in the original scaling) at the bottom of the hindered settling zone. The top of the gelled zone is now at $Z^{\prime}=0$.

The main observation is that even numbered cases (corresponding to faster densification $A=0.01 \mathrm{~s}^{-1}$ or equivalently $\alpha=2.125 \times 10^{-5}$ ) give much more compact gelled zones than odd numbered cases (slower densification $A=0.001 \mathrm{~s}^{-1}$ or equivalently $\left.\alpha=2.125 \times 10^{-6}\right)$ : this reflects the fact that the densification weakens the weight bearing strength of the suspension and thereby promotes gel collapse.

Another observation is that the systems in Figure 7 (smaller $Q_{s}$ and hence higher $\phi_{u}$ ) give substantially taller gelled zones than those in Figure 6 (larger 
$Q_{s}$ and hence lower $\phi_{u}$ ). This reflects the very sharp increase in the weight bearing strength of the suspension with increasing $\phi$.

A similar trend is also seen by comparing sub-plot ' $b$ ' with sub-plot ' $a$ ' in each of these two figures. These compare states with different degrees of densification upon entering the gelled suspension bed, i.e. different $T_{\text {res }}$, but the densification level also affects $Q_{u}$ for any given $Q_{s}$, and hence affects $\phi_{u} \equiv Q_{u} / Q_{s}$. Cases 3-4 in Figure 6 attain a higher underflow solids fraction than their counterparts Cases 1-2 (as indeed Table 1 makes clear) and have a correspondingly taller gelled suspension zone. The effect is however partly offset by Cases 3-4 having a higher gel point than Cases 1-2 (again see Table 1) meaning they also enter the gelled zone with a higher solids fraction.

Figure 6 and Figure 7 also (whereever possible) compare the gelled zone $\phi$ vs $Z^{\prime}$ profiles incorporating aggregate densification with undensified and fully densified counterparts. In order to perform a 'like-for-like' comparison in the gelled suspension zone, the $Q_{u}$ and $\phi_{u}$ values for each of Cases 1-2, $3-4,5-6$ and $7-8$ have been retained in the computations ${ }^{4}$. The qualification 'whereever possible' mentioned above is important: in fact, it is not always possible to implement these like-for-like comparisons, as is explained below.

Comparing with an undensified system relies on the undensified gel point $\phi_{g}$ being significantly greater than the $\phi$ value that gives the local minimum

\footnotetext{
${ }^{4}$ This 'like-for-like' comparison procedure is subtly different from what is presented in Table 1 within which fluxes are set by the hindered settling zone, and so changing the level of densification involves changing the values of $Q_{u}$ and $\phi_{u}$.
} 
on the undensified flux curve. This ensures that, on a set of flux curves such as Figure 3, the undensified flux evaluated at $\phi_{g}$ not only exceeds the local minimum flux on the undensified curve, but is also at least as large as the local minimum on the densified flux curve. Otherwise the undensified gelled system cannot attain the target $Q_{u}$ that (to ensure a like-for-like comparison) is set here by the densified ungelled system. It turns out that for the data presented here it is only possible to do a like-for-like comparison with an undensified system in Figure 6a. The result is as expected: the undensified suspension, which possesses greater weight bearing strength than its densified counterparts, leads to a taller gelled suspension bed.

A like-for-like comparison of the gelled suspension zone with a fully densified system requires that the target underflow solids fraction $\phi_{u}$ be at least as large as the fully densified gel point. The comparison cannot be done in Figure 6 but is possible in Figure 7. As expected the fully densified gelled zone is smaller than any analogous gelled zones that are only partially densified. Particularly in Figure 7a, the gelled zone at full densification is very thin indeed, primarily due to the fact that the fully densified $\phi_{g}$ is exceedingly close to $\phi_{u}$.

Figure 8 shows the combined solids fraction profile for the hindered settling region and gelled bed region taken together. Cases 1-4 are plotted and, so as to collapse data for odd and even numbered cases within the hindered settling region, the plot is given in terms of $\phi$ vs $Z$ (the same scaling as in Figure 5). The figure makes it obvious that height is sometimes dominated 
by the hindered settling region (e.g. Case 3) but sometimes dominated by the gelled bed region (e.g. Case 2). It turns out that Cases 5-8, although not plotted here, tend to be dominated by the gelled bed region. The other observation from the figure is that for Cases 1-4 there is a noticeable jump in solids fraction between the bottom of the hindered settling region (solids fraction $\phi_{\text {loc min }}$ ) and the top of the gelled bed (solids fraction $\phi_{g}$ ): the case of interest in Figure 8 has the same suspension flux as in Figure 4a, and $\phi_{g}$ invariably exceeds $\phi_{l o c}$ min in Figure 4a. This jump from $\phi_{l o c}$ min and $\phi_{g}$ can in certain cases exceed the variation in solids fraction across either the hindered settling region and/or the gelled bed. The size of the jump turns out to be much smaller at the lower suspension flux in Cases 5-8 though (as can also be noted in Table 1 and Figure 4b).

This concludes the discussion of solids fraction profiles. Section 5.3 below considers instead more global height and residence time data.

\subsection{Residence times and heights of the hindered and gelled suspension zones}

Recall that $T_{\text {res }}$ denotes the residence time in the hindered settling zone. The symbol $T_{\text {res, } b}$ is used to denote the residence time in the gelled suspension bed underneath the hindered settling zone, and $T_{\text {res }}^{\text {total }}$ is defined as the sum of $T_{\text {res }}$ and $T_{\text {res,b }}$. Recall also that the origin of the $Z$ coordinate system is placed at the top of the hindered zone, and that the coordinate location $Z=Z_{h}$ is at bottom of the hindered zone. Suppose further that the bottom of the gelled suspension bed is at coordinate location $Z=Z_{h}+Z_{b}$. Both $Z_{h}$ 
and $Z_{b}$ are negative quantities by definition, the sign convention being that $Z$ is measured upwards. Moreover $Z^{\text {total }}$ is taken to be $Z_{h}+Z_{b}$. Table 2 gives $T_{\text {res }}, T_{\text {res }, b}, T_{\text {res }}^{\text {total }},\left|Z_{h}\right|,\left|Z_{b}\right|$ and $\left|Z^{\text {total }}\right|$ for each of Cases 1-8.

In Cases 1-2, times $T_{\text {res,b}}$ spent in the gelled suspension bed are at least as long as times $T_{\text {res }}$ spent in the hindered settling zone. Meanwhile in Cases 3-4 the situation is reversed, times $T_{\text {res }}$ in the hindered settling zone (a parameter that is set) are at least as long as times $T_{r e s, b}$ in the gelled suspension bed (a value that is computed). Cases 5-8, which access much higher solids fractions in the gelled suspension bed than any of Cases 14 , have residence times completely dominated by the gelled zone $T_{r e s, b}$, with only a comparatively small contribution $T_{\text {res }}$ from the hindered settling zone.

Similar considerations apply to the data for thickener heights. In Cases 58 for instance, heights tend to be dominated by the gelled bed contribution $\left|Z_{b}\right|$ with a small contribution from the hindered settling zone $\left|Z_{h}\right|$, albeit the dominance is not quite so strong as for residence times: this is a reflection of the solids moving more slowly in the gelled suspension bed than in the hindered settling zone. Cases 3-4 have somewhat taller hindered zones $\left|Z_{h}\right|$ than gelled suspension beds $\left|Z_{b}\right|$. Case 2 meanwhile has a somewhat taller gelled suspension bed $\left|Z_{b}\right|$ than hindered settling zone $\left|Z_{h}\right|$. Case 1 is an exception: although slightly more time is spent in the gelled suspension bed $T_{r e s, b}$ than in the hindered settling zone $T_{\text {res }}$, the hindered settling zone is slightly taller than the gelled suspension bed $\left(\left|Z_{h}\right|>\left|Z_{b}\right|\right)$. Again this reflects the higher settling speed in the hindered settling zone. 
One counterintuitive feature of Table 2 is that even numbered cases (corresponding to faster densification rates) appear to have taller gelled suspension beds $\left|Z_{b}\right|$ and longer residence times $T_{r e s, b}$ than odd numbered cases (slower densification). This is an artifact of scaling distances and times using scales that themselves involve the densification rate parameter. Recall that in cases with densification rate $A=0.01 \mathrm{~s}^{-1}$ (even numbered cases), 1 unit of dimensionless time corresponds to $100 \mathrm{~s}$ and 1 unit of dimensionless distance corresponds to $8.277 \mathrm{~m}$, but these units of time and distance are increased tenfold when $A=0.001 \mathrm{~s}^{-1}$ (odd numbered cases). Whilst such scalings are useful for collapsing data in the hindered zone, in the gelled suspension bed it is more intuitive (see section 2.3) to rescale the dimensionless distances and times by dividing through by a factor $\alpha$. In that situation, 1 unit of (rescaled) dimensionless time and of (rescaled) dimensionless distance correspond respectively to $2.125 \times 10^{-3} \mathrm{~s}$ and $1.758 \times 10^{-4} \mathrm{~m}$, applicable both to odd and even numbered cases.

Such a rescaling is done in Table 3 with the distance scales in particular now corresponding to those shown in Figure 6 and Figure 7 . This rescaling demonstrates as expected that odd numbered cases (slower densification) do indeed give taller gelled suspension beds, and longer residence times within those beds, than even numbered cases (faster densification).

Table 3 also includes whereever possible like-for-like comparisons with undensified and fully densified systems. As expected undensified systems (arbitrarily slow densification) give the tallest gelled beds and longest resi- 
dence times, whereas fully densified systems (arbitrarily rapid densification) give the most compact gelled beds and shortest residence times.

\section{Conclusions}

A technique has been presented for analysing suspension behaviour in a continuous thickener where the suspension undergoes aggregate densification due to the action of rakes, thereby affecting the suspension rheology and making it easier to dewater. The description of the aggregate densification process is at present empirical: it involves a densification rate parameter that is not itself predicted by the theory. Nonetheless once this parameter is specified, thickener designs can be computed. The technique presented allows matching a Kynch theory based approach (applicable to the hindered settling zone higher up in the thickener) to a Buscall and White based approach (applicable to the gelled suspension zone lower down). The matching technique assumes that the solids fraction at the bottom of the hindered settling zone, which corresponds to a local minimum of a solids flux vs solids fraction curve, is less than the suspension gel point. Under these circumstances it is the hindered settling zone (and not the gelled suspension zone) that determines the solid flux through the suspension.

The model is most easily parameterised in terms of the residence time spent in the hindered settling zone. Depending on the suspension flux that is imposed, it can happen that the solids fraction at the bottom of the hindered zone is invariably less than the suspension gel point, or it might be the case 
that the hindered zone solids fraction is predicted only to remain less than the suspension gel point for a restricted range of hindered settling zone residence times (in which case the transition to the gelled state must occur during that restricted range of times). The smaller the suspension flux, the more likely it is that the range of hindered settling zone residence times will become restricted.

Once hindered settling zone residence times are specified, the model predicts the residence time in a gelled suspension zone beneath the hindered settling zone. It also predicts the heights of each zone. Depending on the parameters selected for the model it is possible to have more time and more height in either the hindered settling zone or in the gelled suspension zone. Smaller suspension fluxes tend to make the gelled suspension zone grow relative to the hindered settling zone.

Different rates of raking the suspension can lead to different rates of densification. The effect of densification rate can be 'scaled out' of the hindered settling zone (i.e. data at different densification rates can be collapsed together by a suitable scaling selecting distance and time units that vary inversely with the densification rate), whereas for the gelled suspension, faster densification leads to a more compact gelled suspension bed.

\section{Acknowledgements}

PG acknowledges sabbatical stay funding from CONICET Argentina res. no. $2218 / 13$. 


\section{References}

Boger, D. V., 2009. Rheology and the resource industries. Chem. Engng Sci. $64,4525-4536$.

Bürger, R., Concha, F., 1998. Mathematical model and numerical simulation of the settling of flocculated suspensions. Int. J. Multiphas. Flow 24, 10051023.

Buscall, R., White, L. R., 1987. The consolidation of concentrated suspensions: Part 1. The theory of sedimentation. J. Chem. Soc. - Faraday Trans. 83, 873-891.

Bustos, M. C., Concha, F., Bürger, R., Tory, E. M., 1999. Sedimentation and Thickening: Phenomenological Foundation and Mathematical Theory. Kluwer Academic, Dordrecht, Netherlands.

Channell, G. M., Miller, K. T., Zukoski, C. F., 2000. Effects of microstructure on the compressive yield stress. AIChE J. 46, 72-78.

Diehl, S., 2001. Operating charts for continuous sedimentation 1: Control of steady states. J. Eng. Math. 41, 117-144.

Diehl, S., 2008. The solids-flux theory - confirmation and extension by using partial differential equations. Water Res. 42, 4976-4988.

Diehl, S., 2012. Shock-wave behaviour of sedimentation in wastewater treatment: A rich problem. In: Åström, K., Persson, L.-E., Silvestrov, S. D. 
(Eds.), Analysis for Science, Engineering and Beyond. Vol. 6. Springer Proceedings in Mathematics, Berlin, pp. 175-214.

Farrow, J. B., Johnston, R. R. M., Simic, K., Swift, J. D., 2000. Consolidation and aggregate densification during gravity thickening. Chem. Engng J. 80, $141-148$.

Fitch, B., 1966. Current theory and thickener design. Ind. Eng. Chem. 58, $18-28$.

Gladman, B. R., Rudman, M., Scales, P. J., 2010. The effect of shear on gravity thickening: Pilot scale modelling. Chem. Engng Sci. 65, 4293-4301.

Grassia, P., Usher, S. P., Scales, P. J., 2008. A simplified parameter extraction technique using batch settling data to estimate suspension material properties in dewatering applications. Chem. Engng Sci. 63, 1971-1986.

Grassia, P., Zhang, Y., Martin, A. D., Usher, S. P., Scales, P. J., Crust, A., Spehar, R., 2014. Effects of aggregate densification upon thickening of Kynchian suspensions. Chem. Engng Sci. 111, 56-72.

Heath, A. R., Bahri, P. A., Fawell, P. D., Farrow, J. B., 2006. Polymer flocculation of calcite: Relating the aggregate size to the settling rate. AIChE J. 52, 1987-1994.

Jones, H., Boger, D. V., 2012. Sustainability and waste management in the resource industries. Ind. Eng. Chem. Res. 51, 10057-10065. 
Kynch, G. J., 1952. A theory of sedimentation. Trans. Faraday Soc. 48, 166176.

Landman, K. A., White, L. R., 1994. Solid/liquid separation of flocculated suspensions. Adv. Colloid \& Interface Sci. 51, 175-246.

Landman, K. A., White, L. R., Buscall, R., 1988. The continuous-flow gravity thickener: Steady state behavior. AIChE J. 34, 239-252.

Lester, D. R., Usher, S. P., Scales, P. J., 2005. Estimation of the hindered settling function $R(\phi)$ from batch-settling tests. AIChE J. 51, 1158-1168.

Martin, A. D., 2004. Optimisation of clarifier-thickeners processing stable suspensions for turn-up/turn-down. Water Res. 38, 1568-1578.

Matsche, N., Winkler, S., Prendl, L., Dornhofer, K., Wandl, G., 2002. Treatment of easily biodegradable wastewater avoiding bulking sludge. Water Sci. Technol. 46 (12), 503-506.

Pere, J., Alen, R., Viikari, L., Eriksson, L., 1993. Characterization and dewatering of activated-sludge from the pulp and paper-industry. Water Sci. Technol. 28 (1), 193-201.

Spehar, R., Kiviti-Manor, A., Fawell, P., Usher, S. P., Rudman, M., Scales, P. J., 2014. Aggregate densification in the thickening of flocculated suspensions in an un-networked bed. Chem. Engng Sci. 122, 585-595. 
Talmage, W. P., Fitch, E. B., 1955. Determining thickener unit areas. Ind. Eng. Chem. 47, 38-41.

Usher, S. P., Scales, P. J., 2005. Steady state thickener modelling from the compressive yield stress and hindered settling function. Chem. Engng J. $111,253-261$.

Usher, S. P., Spehar, R., Scales, P. J., 2009. Theoretical analysis of aggregate densification: Impact on thickener performance. Chem. Engng J. 151, 202208.

van Deventer, B. B. G., Usher, S. P., Kumar, A., Rudman, M., Scales, P. J., 2011. Aggregate densification and batch settling. Chem. Engng J. 171, $141-151$

Zhang, Y., Grassia, P., Martin, A. D., Usher, S. P., Scales, P. J., 2015. Mathematical modelling of batch sedimentation subject to slow aggregate densification. Chem. Engng Sci. 128, 54-63.

Zhang, Y., Martin, A., Grassia, P., 2013a. Mathematical modelling of timedependent densified thickeners. Chem. Engng Sci. 99, 103-112.

Zhang, Y., Martin, A., Grassia, P., 2013b. Prediction of thickener performance with aggregate densification. Chem. Engng Sci. 101, 346-358. 


\begin{tabular}{|c|c||c|c|c|c|c|}
\hline & $Q_{s}$ & $T_{\text {res }}$ & $Q_{u}$ & $\phi_{u}$ & $\phi_{\text {loc } \min }$ & $\phi_{g}$ \\
\hline unden & 0.0034 & $\mathrm{~N} / \mathrm{A}$ & 0.000364 & 0.1071 & 0.0728 & 0.1 \\
\hline Cases 1-2 & 0.0034 & 0.05 & 0.000370 & 0.1088 & 0.0741 & 0.1014 \\
\hline Cases 3-4 & 0.0034 & 0.3 & 0.000397 & 0.1166 & 0.0798 & 0.1082 \\
\hline Full den & 0.0034 & $\mathrm{~N} / \mathrm{A}$ & 0.000513 & 0.1509 & 0.1049 & 0.1372 \\
\hline unden & 0.0015 & $\mathrm{~N} / \mathrm{A}$ & 0.000205 & 0.1367 & 0.0982 & 0.1 \\
\hline Cases 5-6 & 0.0015 & 0.05 & 0.000208 & 0.1390 & 0.0999 & 0.1014 \\
\hline Cases 7-8 & 0.0015 & 0.3 & 0.000222 & 0.1483 & 0.1078 & 0.1082 \\
\hline Full den & 0.0015 & $\mathrm{~N} / \mathrm{A}$ & 0.000285 & 0.19 & 0.1420 & 0.1372 \\
\hline
\end{tabular}

Table 1: Thickener operating parameters for Cases 1-8 in terms of dimensionless suspension flux $Q_{s}$ and dimensionless residence time in the hindered settling zone $T_{\text {res }}$ (which governs the extent of aggregate densification). Comparisons with undensified and fully densified cases (for the same suspension flux $Q_{s}$ ) are also shown. Solids flux $Q_{u}$, underflow solids fraction $\phi_{u}$, the 'local minimum' solids fraction $\phi_{l o c}$ min (that delivers the required solids flux being a local minimum of the flux curve at time $\left.T_{r e s}\right)$ and gel point $\phi_{g}$ are determined. Odd numbered cases correspond to aggregate densification rate $A=0.001 \mathrm{~s}^{-1}$ (equivalently in dimensionless form, $\alpha=2.125 \times 10^{-6}$ ) whilst even numbered cases correspond to $A=0.01 \mathrm{~s}^{-1}$, (equivalently $\alpha=2.125 \times 10^{-5}$ ), but the effect of this parameter is scaled out of the hindered settling zone, although it remains relevant in the gelled suspension zone. In almost all cases shown the constraint $\phi_{l o c} \min <\phi_{g}$ is satisfied, the only exception being the fully densified case with $Q_{s}=0.0015$ (indicating that the suspension could not attain full densification without gelling at this particular $Q_{s}$ value). 


\begin{tabular}{|c|c|c|c|}
\hline & $T_{\text {res }}$ & $T_{\text {res }, b}$ & $T_{\text {res }}^{\text {total }}$ \\
\hline Case 1 & 0.05 & 0.0587 & 0.1087 \\
\hline Case 2 & 0.05 & 0.2044 & 0.2544 \\
\hline Case 3 & 0.3 & 0.0769 & 0.3769 \\
\hline Case 4 & 0.3 & 0.2802 & 0.5802 \\
\hline Case 5 & 0.05 & 0.8282 & 0.8782 \\
\hline Case 6 & 0.05 & 2.3423 & 2.3923 \\
\hline Case 7 & 0.3 & 1.0409 & 1.3409 \\
\hline Case 8 & 0.3 & 4.1924 & 4.4924 \\
\hline \hline & $\left|Z_{h}\right|$ & $\left|Z_{b}\right|$ & $\left|Z^{\text {total }}\right|$ \\
\hline Case 1 & 0.000276 & 0.000207 & 0.000483 \\
\hline Case 2 & 0.000276 & 0.00072 & 0.000996 \\
\hline Case 3 & 0.00193 & 0.00028 & 0.00221 \\
\hline Case 4 & 0.00193 & 0.00099 & 0.00292 \\
\hline Case 5 & 0.000114 & 0.001476 & 0.00159 \\
\hline Case 6 & 0.000114 & 0.003946 & 0.00406 \\
\hline Case 7 & 0.000775 & 0.001855 & 0.00263 \\
\hline Case 8 & 0.000775 & 0.006975 & 0.00775 \\
\hline
\end{tabular}

Table 2: For Cases 1-8, dimensionless solids residence times spent in the hindered settling zone $T_{\text {res }}$ (a parameter that is set), residence times in the gelled suspension region $T_{r e s, b}$ (a parameter that is computed), and also the sum of these, i.e. the total solids residence times, $T_{r e s}^{\text {total }}$. Also shown are heights required for the hindered settling zone $\left|Z_{h}\right|$, gelled suspension zone $\left|Z_{b}\right|$, and total thickener heights $\left|Z^{\text {total }}\right| \equiv\left|Z_{h}\right|+\left|Z_{b}\right|$. Recall that the (dimensionless) suspension flux, $Q_{s}$ equals 0.0034 for Cases 1-4 and 0.0015 for Cases 5-8. Recall also that the (dimensional) densification rate parameters are $A=0.001 \mathrm{~s}^{-1}$ for odd numbered cases and $A=0.01 \mathrm{~s}^{-1}$ for even numbered cases; equivalently $\alpha=2.125 \times 10^{-6}$ for odd numbered cases, and $\alpha=2.125 \times 10^{-5}$ for even numbered cases. 


\begin{tabular}{|c|c|c|c|c|}
\hline & $Q_{u}$ & $\phi_{u}$ & $\left|Z_{b} / \alpha\right|$ & $T_{r e s, b} / \alpha$ \\
\hline Unden & 0.000370 & 0.1088 & 153.5 & 43009 \\
\hline Case 1 & 0.000370 & 0.1088 & 97.4 & 27623.5 \\
\hline Case 2 & 0.000370 & 0.1088 & 33.9 & 9618.8 \\
\hline Full den & N/A & N/A & N/A & N/A \\
\hline \hline Unden & N/A & N/A & N/A & N/A \\
\hline Case 3 & 0.000397 & 0.1166 & 131.8 & 36188.2 \\
\hline Case 4 & 0.000397 & 0.1166 & 46.6 & 13185.9 \\
\hline Full den & N/A & N/A & N/A & N/A \\
\hline \hline Unden & N/A & N/A & N/A & N/A \\
\hline Case 5 & 0.000208 & 0.1390 & 694.6 & 389741 \\
\hline Case 6 & 0.000208 & 0.1390 & 185.7 & 110226 \\
\hline Full den & 0.000208 & 0.1390 & 37.7 & 25025 \\
\hline \hline Unden & N/A & N/A & N/A & N/A \\
\hline Case 7 & 0.000222 & 0.1483 & 872.9 & 489835 \\
\hline Case 8 & 0.000222 & 0.1483 & 328.2 & 197289 \\
\hline Full den & 0.000222 & 0.1483 & 233.5 & 149676 \\
\hline
\end{tabular}

Table 3: Rescaled gelled suspension bed heights $\left|Z_{b} / \alpha\right|$ and rescaled solids residence times $T_{\text {res,b }} / \alpha$ spent in the consolidation region for Cases $1-8$. Odd numbered cases have smaller $\alpha$ than even numbered ones $\left(\alpha=2.125 \times 10^{-6}\right.$ vs $\left.\alpha=2.125 \times 10^{-5}\right)$, i.e. they correspond to slower aggregate densification. Cases are compared (whereever possible) with an undensified system and a fully densified system operated at the same solids flux $Q_{u}$ and the same underflow solids fraction $\phi_{u}$. In many cases, the undensified data are omitted, as the undensified system cannot simultaneously deliver the target underflow solids flux and target underflow solids volume fraction. In certain of the fully densified cases, data are again omitted, because no gelled suspension bed is required for a fully densified system at the underflow solids volume fraction in question. 


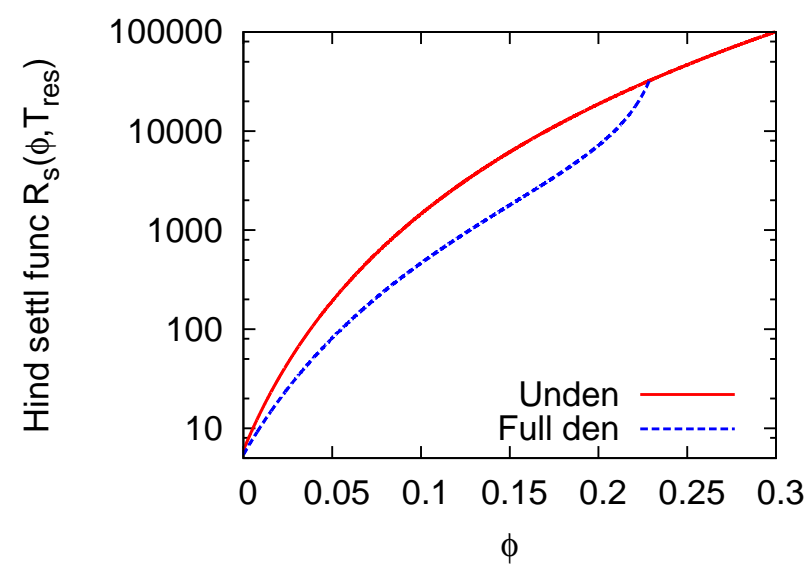

Figure 1: Dimensionless hindered settling function $R_{s}$ (defined as $R_{s} \equiv R / R_{\text {Stokes }, 0}$ ) vs solids fraction $\phi$ for the undensified state $\left(T_{\text {res }}=0\right)$ and also for the fully densified state $\left(T_{\text {res }} \rightarrow \infty\right)$. Values of $R_{s}$ vs $\phi$ corresponding to intermediate levels of aggregate densification would lie between the two curves shown. Note that for solids fractions $\phi$ in excess of $\phi_{a g g}$, the undensified hindered settling function is recovered, since aggregates then fill space and are interlinked and interpenetrating, and so are no longer discrete entities. Such large $\phi$ values are however beyond the range of solids fractions of interest in the present work. 


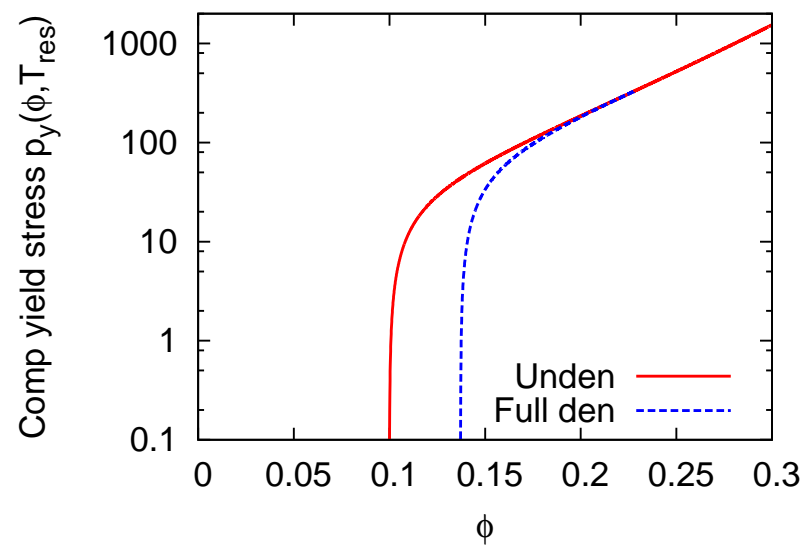

Figure 2: Dimensionless compressive yield stress $p_{y}$ (defined as $p_{y} \equiv P_{y} / a_{0}$ ) vs solids fraction $\phi$ for the undensified state $\left(T_{\text {res }}=0\right)$ and for the fully densified state $\left(T_{\text {res }} \rightarrow \infty\right)$. Values of $p_{y}$ vs $\phi$ corresponding to intermediate levels of aggregate densification would lie between the two curves shown. For solids fractions $\phi$ in excess of $\phi_{\text {agg }}$, the undensified hindered settling function is recovered (Channell et al., 2000), although that is beyond the range of solids fractions of interest in the present work. 


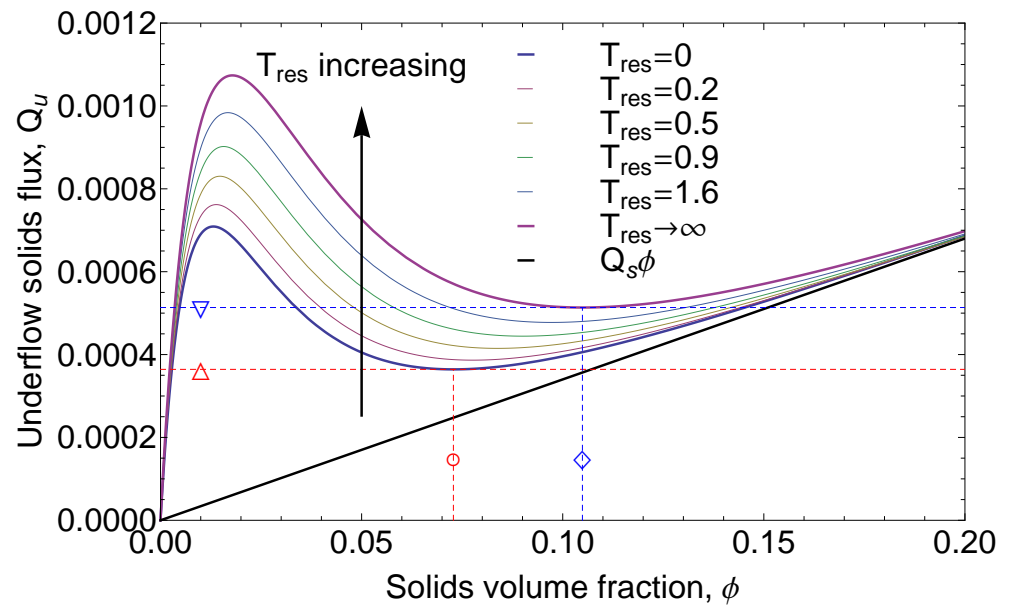

Figure 3: Dimensionless solids flux $Q_{u} \equiv Q_{s} \phi+Q_{f s}$ vs solids fraction $\phi$ in the hindered settling zone of the suspension for a suspension flux $Q_{s}=0.0034$ and various residence times $T_{\text {res }}$ (corresponding to various different levels of aggregate densification achieved in the hindered zone). Interest focusses on the local minimum for each curve. Increasing the value of $T_{\text {res }}$ not only increases the value of the local minimum (indicated by horizontal dashed lines), but also increases the solids fraction $\phi$ corresponding to the minimum (dashed vertical lines). The aggregate diameter ratio $D_{\text {agg }}$ that governs the level of densification varies from unity at $T_{\text {res }}=0$ to 0.9 as $T_{\text {res }} \rightarrow \infty$. 

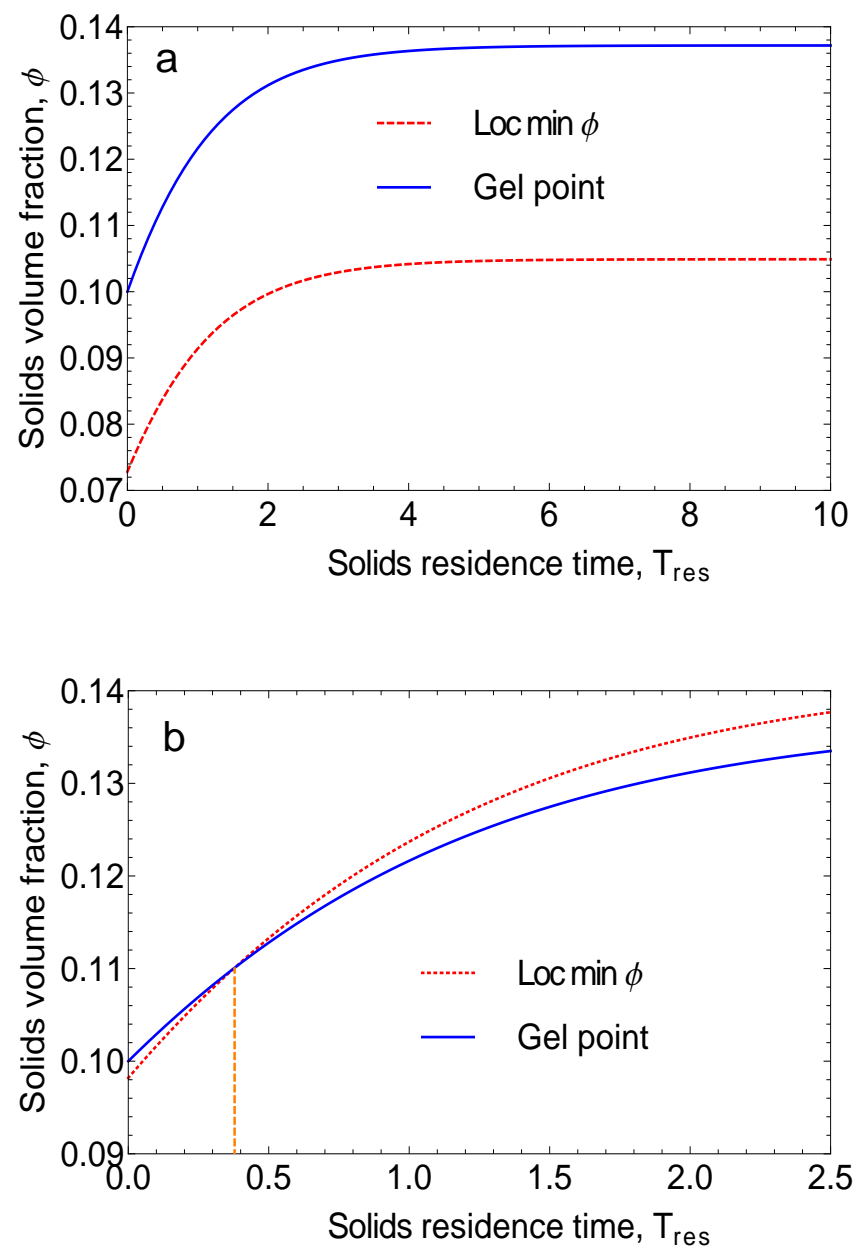

Figure 4: The solids fraction $\phi_{l o c} \min$ (corresponding to the local minimum of the $Q_{u}$ vs $\phi$ curve, equation (14)) and the gel point $\phi_{g}$ both plotted vs residence time $T_{r e s}$ in the hindered settling zone. In the sub-plot labelled 'a', the suspension flux is $Q_{s}=0.0034$ and $\phi_{\text {loc } \min }<\phi_{g}$ at all times. In the sub-plot labelled 'b', the suspension flux is $Q_{s}=0.0015$ and the curves intersect at $T_{r e s} \approx 0.378$. Matching the hindered settling zone to the gelled suspension bed is only possible for times up to that intersection point, so (for sub-plot ' $b$ ') the suspension needs to have gelled by that time. 

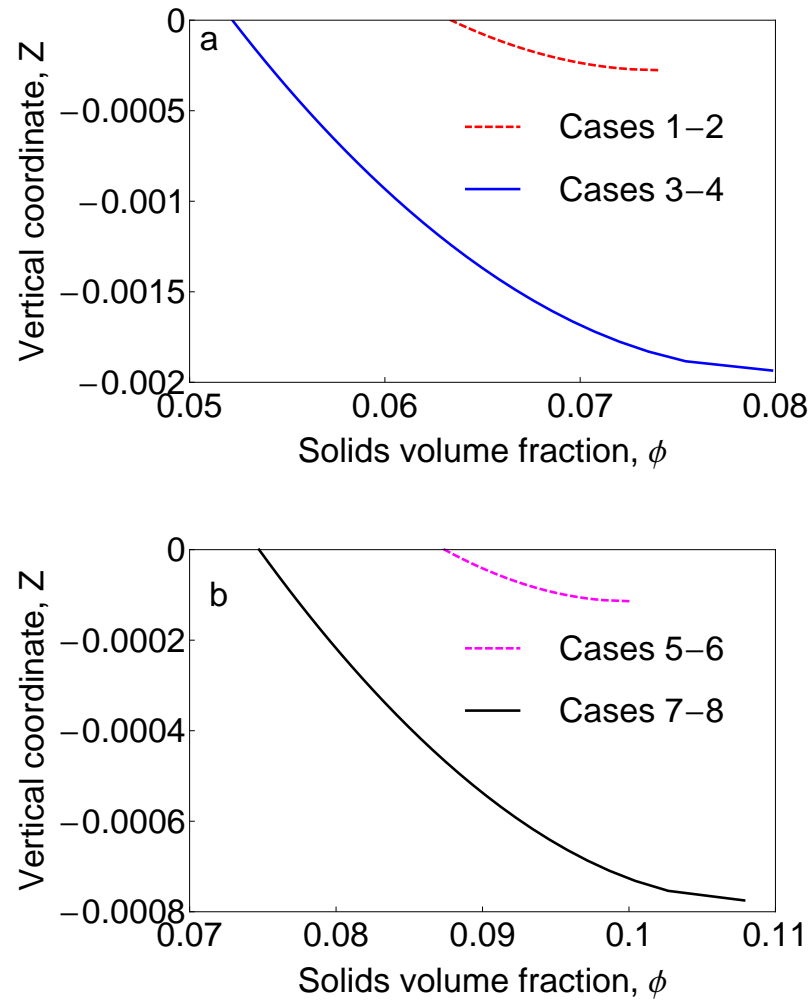

Figure 5: Profiles of the solids volume fractions $\phi$ vs dimensionless position $Z$ in the hindered settling region. Note that there is no difference between adjacent odd and even numbered cases, since they only differ in their value of the densification rate parameter, $A$, but the effect of this parameter has been scaled out of the hindered settling region. The sub-plots labelled 'a' and 'b' are plotted using suspension fluxes $Q_{s}=0.0034$ and $Q_{s}=0.0015$ respectively. Recall that $T_{\text {res }}$ (the dimensionless residence time that governs the extent of aggregate densification at the bottom of the hindered settling zone) equals 0.05 for Cases 1-2 and Cases 5-6, and equals 0.3 for Cases 3-4 and Cases 7-8. 

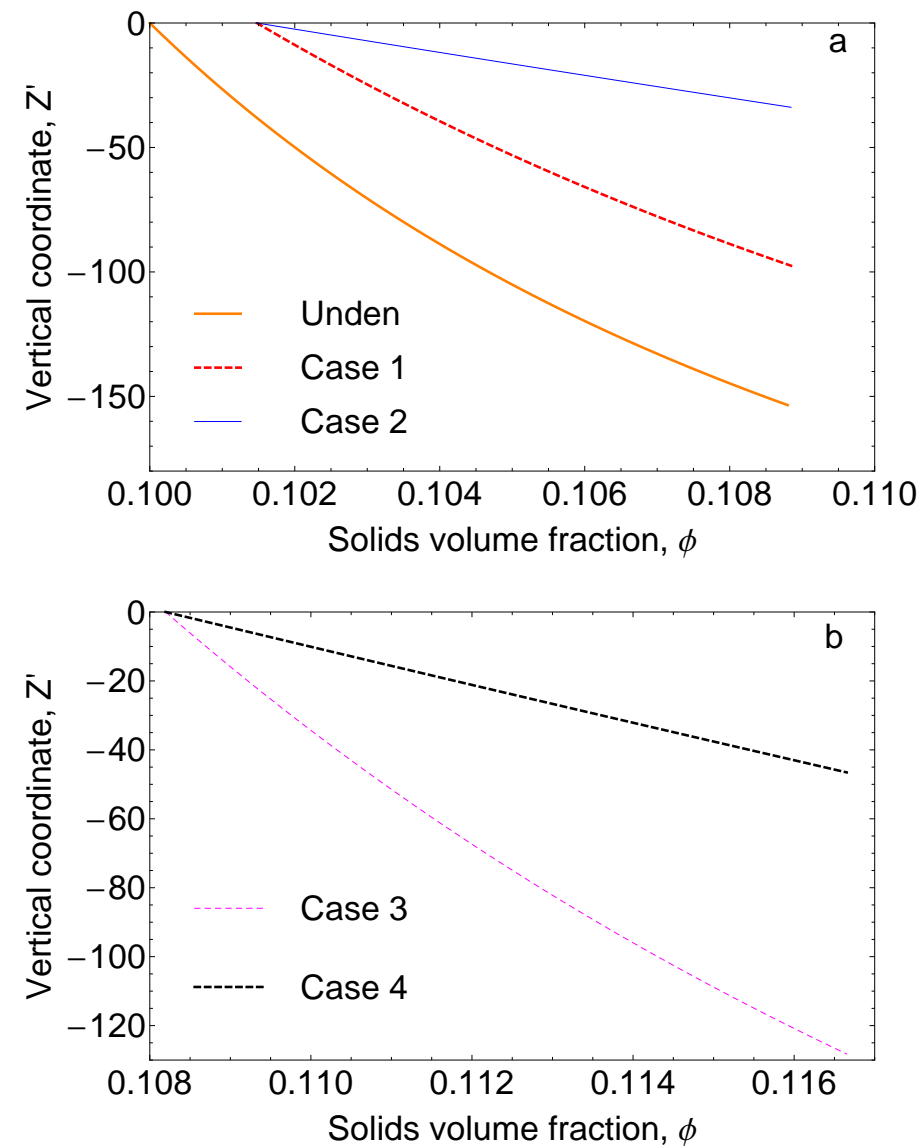

Figure 6: Solids volume fraction profiles determined in the gelled suspension zone in rescaled coordinates $\phi$ vs $Z^{\prime}$, and the comparison with the undensified case (where applicable). No comparison is given with the fully densified case (because, for the parameter values given, the fully densified case does not gel at the underflow solids volume fraction in question). Recall that the suspension flux is $Q_{s}=0.0034$ in all these cases. 

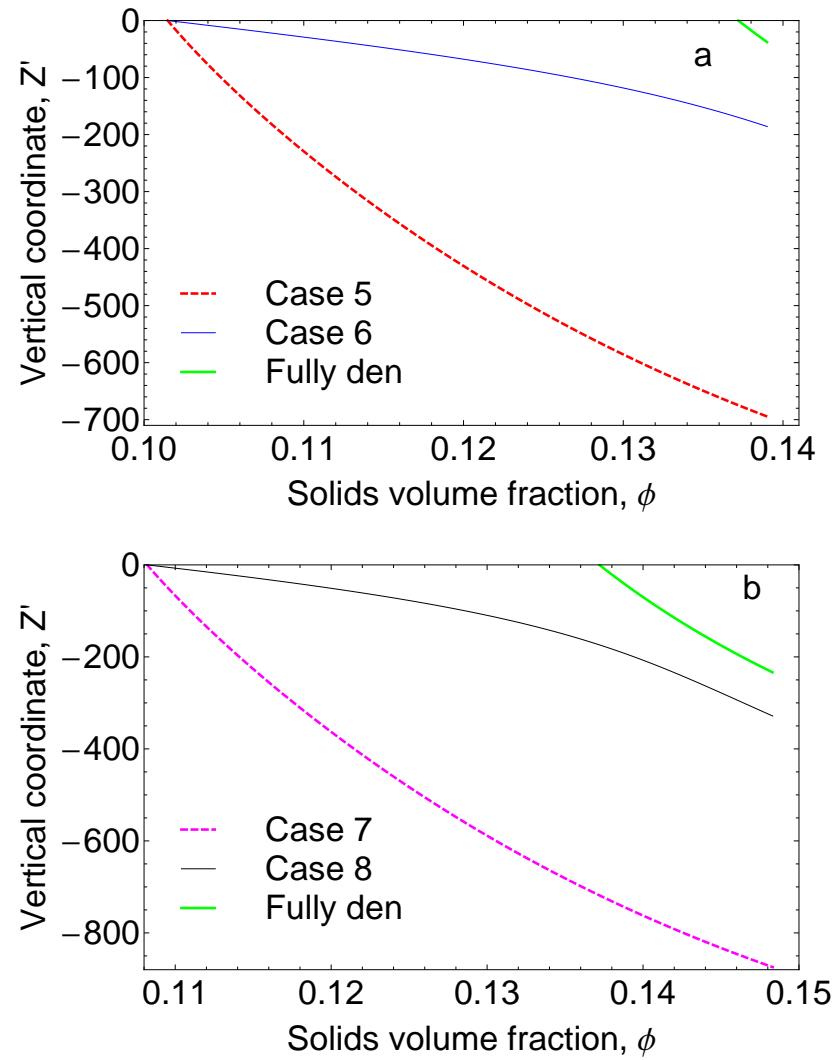

Figure 7: Solids volume fraction profiles determined in the gelled suspension region in rescaled coordinates $\phi$ vs $Z^{\prime}$ and comparisons with fully densified cases. No comparison is given with the undensified case, since the undensified case cannot simultaneously attain the same underflow solids flux and the same underflow solids volume fraction as these densified ones. Recall that the specified suspension flux is $Q_{s}=0.0015$ in these cases. 

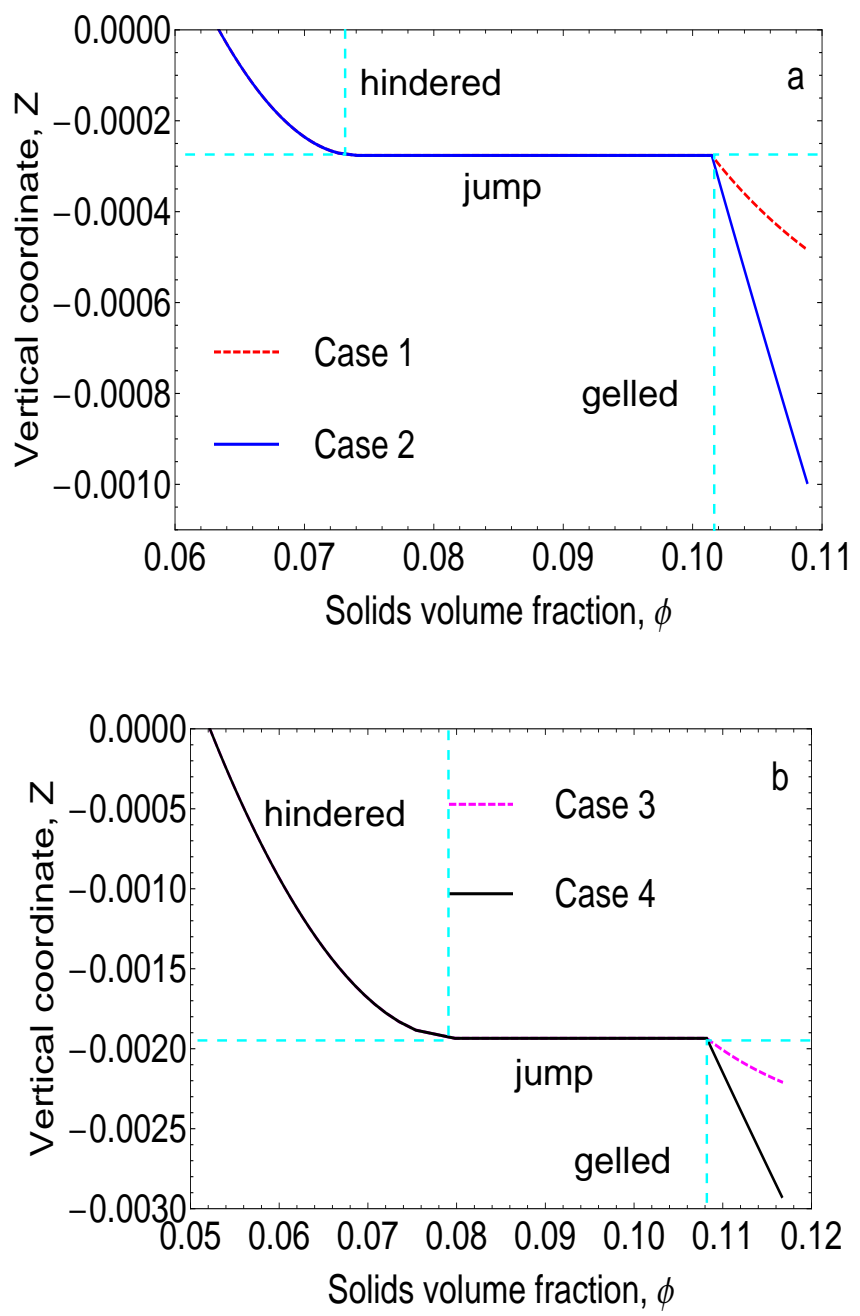

Figure 8: Solids volume fraction profiles $\phi$ vs dimensionless position $Z$ in both the hindered settling region above and in the gelled suspension region below with a jump in solids fraction between the two regions. Cases 1-2 are shown in sub-plot 'a', cases 3-4 are shown in sub-plot 'b', and all of these have suspension flux $Q_{s}=0.0034$. 